\title{
PRECIOS DE MERCADO COMO MEDIO DE VALORACIÓN DE LOS BENEFICIOS AMBIENTALES DE LAS RESERVAS MARINO COSTERAS DEL ECUADOR
}

\author{
MARKET PRICES AS A TOOL TO VALUE ENVIRONMENTAL SERVICES OF \\ COASTAL-MARINE RESERVES OF ECUADOR
}

ANA GABRIELA CADENA ${ }^{1}$, DARÍO ECHEVERRÍA ${ }^{1}$, LISSETH FIERRRO ROMERO ${ }^{1}$, KARLA VARGAS ${ }^{1}$, FABIÁN RODRÍGUEZ ESPINOSA ${ }^{2,3}$

${ }^{1}$ CARRERA DE INGENIERÍA GEOGRÁFICA Y DEL MEDIO AMBIENTE, UNIVERSIDAD DE LAS FUERZAS ARMADAS ESPE, ECUADOR. agcadena1@espe.edu.ec, djecheverria@espe.edu.ec, lnfierro@espe.edu.ec, kalysole1993@gmail.com.

2 DEPARTAMENTO DE CIENCIAS DE LA TIERRA, UNIVERSIDAD DE LAS FUERZAS ARMADAS ESPE, SANGOLQUÍ, ECUADOR. ffrodriguez3@espe.edu.ec

${ }^{3}$ FACULTAD DE ECONOMÍA, PONTIFICIA UNIVERSIDAD CATÓLICA DEL ECUADOR PUCE, QUITO, ECUADOR.

Recibido: 2 de abril de 2018 /Aceptado: 28 de junio de 2018

\section{RESUMEN}

El presente trabajo se realizó para obtener un valor económico de cuatro reservas marino-costeras del Ecuador mediante la aplicación de los métodos de costo de mercado. Dos bienes y servicios ambientales fueron evaluados para estimar el valor económico de las cuatro reservas. La producción de alimento, la producción pesquera de carácter artesanal que está permitida en las áreas protegidas y tienen una superficie asignada para ello en particular fue estimada a través del método de precio de mercado. El método de transferencia de beneficios fue utilizado para la estimación del almacenamiento de carbono sobre la base de la estimación de la biomasa seca de los tipos de bosques naturales existentes en las cuatro reservas. El aporte económico de estas reservas alcanzó 105 millones de dólares, siendo la pesca artesanal con mayor aporte con 100,09 millones. La pesca artesanal da un aporte directo a aproximadamente 6800 familias en las cuatro reservas que tuvieron ingresos promedio de 14.720,18 dólares anuales, ingresos que son superiores a los 5.968,98 dólares del PIB per cápita del Ecuador para el año 2016. Las cuatro reservas aportaron con 5,3 millones de dólares en almacenamiento de carbono. Para el almacenamiento de carbono se utilizó el precio de referencia del mercado de carbono del Banco Mundial. El aporte económico de las cuatro reservas marino costeras es relevante si lo comparamos con el presupuesto del Estado (PGE) en 2016 y con el PIB de ese año. Los beneficios económicos de las cuatro reservas marino-costeras representan el 0,35\% del PGE y $0,1 \%$ del PIB para el año 2016. En definitiva los precios de mercado son una buena herramienta para la valoración de los bienes y servicios ambientales de áreas protegidas marino-costeras.

Palabras clave: Valoración económica, precio de mercado, bienes y servicios ambientales.

\begin{abstract}
This study estimated the economic value of environmental good and services in four coastal-marine protected areas in Ecuador using market price method. Two environmental good and services were used to estimate the economic value of these reserves, food and carbon storage. The market price method was used in both environmental services. In case of food services, information from craftsman fishing in the four reserves and estimate carbon storage, a benefit transfer method was applied. The results show an input of 105 million dollars, one hundred of them from craftsman fishing. Craftsman fishing has a direct impact on 6.800 families living in reserves' area, having an annual average income of 14,720.18 dollars. This income is higher than Ecuadorean PIB per capita of 5,968.98 dollars. The four reserves also contribute with
\end{abstract}


5.3 million dollars from carbon storage. We use World Bank carbon market as reference price for carbon storage. The economic benefits of these four coastal-marine protected areas stand for $0.35 \%$ of the GNB (general national budget) and $0.1 \%$ of PIB for 2016. Definitely, market price method is a valid tool to value environmental good and services in coastal-marine protected areas.

Key words: Economic valuation, market price, environmental good and services.

\section{INTRODUCCIÓN}

En las últimas décadas ha surgido la necesidad de mejorar la forma en la que utilizamos los recursos naturales en particular los recursos costeros y marino ya que 3,1 billones de personas dependen directamente del recurso marino (OECD, 2016; Sala et al., 2013). En este contexto, países alrededor del mundo han ampliado o creado reservas marinocosteras. Existen más de 1000 reservas marinas en 87 países (Salm et al., 2000; Silva et al., 1986). Además, se han desarrollado estrategias que buscan más allá de la conservación de estas áreas, para alcanzar una administración sostenible y sustentable de los recursos y servicios marino-costeros (Salgado, 2016; Sala et al., 2013; Sanchirico et al., 2002). Las zonas marino-costeras están entre los ecosistemas de mayor peligro de desaparecer y a la vez son los ecosistemas más críticos por los beneficios y recursos que provee (Salm et al., 2000). Las reservas marino costeras juegan un importante rol en la provisión de alimentos, según Sala et al. (2013) las reservas marino costeras tienen un $21 \%$ más de especies que áreas similares no protegidas; en tamaño, las especies tiene un $28 \%$ mayor tamaño que en áreas similares, y existe una mayor densidad de especies por área. La industria pesquera se puede beneficiar del establecimiento de áreas protegidas en zonas marino costeras, ya que llegan a ser las guarderías naturales para la recuperación de las especies de peces, especialmente comerciales (Xuan and Amstrong, 2016; Hanley et al., 2015; Akhter and Yew, 2013; Sala et al., 2013; Sanchirico et al., 2002; Salm et al., 2000). Adicionalmente, las reservas marino costeras proveen una serie de servicios que benefician a las poblaciones que se encuentran en estas zonas. Entre los servicios se incluye la protección costera contra marejadas, tsunamis e inundaciones, como albergue de biodiversidad y secuestro y almacenamiento de carbono (OECD, 2016). Sin embargo, las presiones por actividades humanas de estos ecosistemas críticos son múltiples y en aumento. Según la OECD (2016), los sistemas de acuacultura marina van a triplicarse en las próximas dos décadas, la extracción industrial de peces se duplicará en el mismo período al igual que las actividades portuarias y de turismo. Según datos de la FAO (2016) la pesca industrial está en niveles biológicamente insostenibles. El 80\% de la contaminación de las zonas marino costera se debe al vertido de fertilizantes y pesticidas en los sistemas agrícolas de las zonas continentales que filtran en el drenaje natural de los ríos que desembocan en los océanos (GESAMP, 2018, 2016; OECD, 2016; Vikas and Dwarakish, 2015; Shahidul Islam and Tanaka, 2004).

Los efectos de las actividades humanas como la contaminación se agravan con los efectos del cambio climático mundial. Uno de los principales efectos es el aumento de la temperatura y acidez de los océanos (Doney et al., 2016, 2009; Bindoff et al., 2007). El 
aumento de la temperatura global afecta tanto a la diversidad de especies como también a los ecosistemas. Según Doney et al. (2016) ya existe una degradación o pérdida de marismas en un 50\%, así como también de los manglares, arrecifes de coral y lechos de algas marinas con un reducción de $30 \%$. La pérdida del hábitat en las costas y océanos afecta directamente a la pesca artesanal como también al turismo uno de las potenciales fuentes de ingresos para las comunidades costeras (OECD, 2016)

Debido al impacto de las actividades humanas en los océanos y zonas costeras, gobiernos alrededor del mundo han desarrollado políticas para conservar estas áreas críticas. Una de las políticas es la creación de áreas marinas de protección para la conservación de su riqueza biológica y de sus hábitats, como también la serie de servicios ecosistémicos que son fundamentales para el bienestar humano (OECD, 2016)

Siguiendo con esta política a nivel global, el Ecuador ha creado 16 reservas marinocosteras incluyendo el Parque Nacional Galápagos y la Reserva Marina Galápagos, la de mayor extensión, que forman parte el Sistema Nacional de Áreas Protegidas SNAP (MAE, 2015a). Las áreas protegidas son de real importancia para la conservación de la biodiversidad en nuestro país teniendo como principal objetivo la conservación de la diversidad biológica y de los recursos genéticos y brindar alternativas de aprovechamiento sustentable de los recursos y la prestación adecuada de bienes y servicios ambientales. Adicionalmente, según la Fundación Futuro Latinoamericano (2011), el Ministerio del Ambiente del Ecuador y la Subsecretaría de Pesca declararon zona de reserva para la reproducción de especies bioacuáticas a la zona comprendida desde la costa continental hasta una milla náutica hacia el mar, que poseen un valor incalculable en cuanto a los beneficios que brindan a la economía nacional y a la sociedad. Debido a lo anterior expuesto, es importante conocer la necesidad de cuales son los servicios económicos que pueden generar con relación a otro tipo de manejo sobre el área; es así que, se han realizado algunos estudios de valoración especialmente en manglares como presenta Rodríguez-Espinosa et al. (2017) en el estudio que analiza el impacto económico del servicio de protección contra marejadas y tsunamis. La mayoría de metodologías de valoración están limitadas por los datos que estén liberados o existentes.

\section{DESCRIPCIÓN GEOGRÁFICA}

Se seleccionaron cuatro reservas marino costeras del Ecuador para estimar el aporte económico y sustentar la decisión política de la creación de estas zonas. Las reservas y áreas protegidas seleccionadas tienen temperaturas promedio anuales muy parecidas, pero por la ubicación de cada una de ellas, los niveles de precipitación varían radicalmente (Tabla 1).

Con relación a la extensión, la Reserva marino-costera Galera-San Francisco es la más grande con $540 \mathrm{~km}^{2}$, las otras tres reservas y refugios son más pequeñas con apenas un área entre 13 y $52 \mathrm{~km}^{2}$ (Tabla1). 
Tabla 1. Áreas seleccionadas de estudio

\begin{tabular}{|c|c|c|c|c|}
\hline Reserva Marina & Área $\left(\mathrm{km}^{2}\right)$ & $\begin{array}{c}\text { Temp. } \\
\text { Prom. }\left({ }^{\circ} \mathrm{C}\right)\end{array}$ & $\begin{array}{c}\text { Precip- } \\
\text { itación }(\mathrm{mm})\end{array}$ & $\begin{array}{c}\text { Línea Cos- } \\
\text { tera }(\mathrm{km})\end{array}$ \\
\hline Galera-San Francisco & 540 & 25 & $500-3000$ & 37 \\
\hline El Pelado & 13,101 & 23,4 & 240 & \\
\hline La Puntilla de Santa Elena & 52,44 & 24 & 155 & \\
\hline Pacoche & 13,56 & 24 & 214 & 15 \\
\hline
\end{tabular}

Fuente: GADs Planes de Desarrollo Territorial. Elaboración: Autores

Los tipos de vegetación varían entre las zonas de reservas marinas seleccionadas, pero predomina el bosque seco costero ecuatoriano, se distinguen seis formaciones vegetales naturales: Bosque siempre verde piemontano de la cordillera de la Costa, Bosque semideciduo de tierras bajas, Bosque deciduo de tierras bajas, Matorral seco de tierras bajas de la Costa, Matorral seco litoral, Espinar litoral. La Reserva Marina GaleraSan Francisco se localiza en la zona crítica de conservación (hotspot) Tumbes-Chocó Magdalena, comprende los bosques húmedos y muy húmedos de la región del Pacífico en Colombia y Esmeraldas en Ecuador, así como los bosques secos de la provincia de Manabí en Ecuador (Luna y Campos, 2008; NAZCA, 2006).

La Reserva Marino Costera Galera-San Francisco se ubica en la península del mismo nombre, ha sido identificado como uno de los cinco bloques de máxima prioridad de conservación y manejo debido a un conjunto de atributos oceanográficos, climáticos y físicos que determinan la composición de comunidades de flora y fauna especial para el Ecuador continental. El área Galera-San Francisco se encuentra en la zona ecológica tropical cálida en donde la variación de la temperatura superficial se mantiene entre los 24 y $25^{\circ} \mathrm{C}$ a lo largo de todo el año, a diferencia del resto del Ecuador que presenta variaciones más amplias y una dominancia de aguas temperadas. El área es una de las tres zonas del país en donde la plataforma continental alcanza su mayor estrechamiento y pendiente. La península Galera-San Francisco (Figura 1a) abarca cinco de los seis tipos de playas que existen en la costa ecuatoriana: playas de arena, de limo, de roca, mixta (arena y roca) y mixta con arrecifes franqueantes de poliquetos. En la zona submareal, el área posee fondos blandos de arena y/o limo, fondos duros de roca y/o laja y rocas sumergidas. Estos ecosistemas soportan una compleja interacción entre los organismos marinos y proporcionan hábitats diferentes para una amplia diversidad de especies (NAZCA, 2006).

Los organismos asociados a estos hábitats son de origen tropical y sus principales representantes constituyen los bosques de gorgonias y otros tipos de corales, existe evidencia de que la mayor población de coral negro del Ecuador se encuentra en esta zona. El área alberga una gran diversidad de especies comprendida por más de 600 especies registradas, representantes como el pulpo y caracoles son parte de la alimentación 
diaria de los pobladores locales. En esta zona se encuentra una gran variedad de especies de peces asociados a fondos rocosos y coralinos, muchos de ellos potencialmente importantes como atractivos turísticos. Existen también varias especies importantes para la industria pesquera como son la corvina de roca, el pargo y el dorado por ello, es de vital importancia cuantificar el valor monetario que tienen esos servicios para poder integrar un plan de manejo y protección del medio ambiente (Peralta, 2001).

La reserva marina "El Pelado", cuyo frente de línea da con la costa del Ecuador continental, ubicándose entre las provincias de Manabí y Santa Elena. La reserva posee varios islotes que enriquecen el paisaje, siendo sitios ideales para el refugio y la anidación de aves marinas, encontrándolo frente a la playa de Ayangue, como lo muestra la Figura 1b. La reserva protege el islote El Pelado, el mar que lo rodea y la línea costera entre las poblaciones de Valdivia San Pedro, Ayangue y Palmar. Su extensión es de 96 hectáreas de zona terrestre y 13.005 hectáreas de zona marina y más de 78 especies de peces. Es considerada como reserva marina al poseer un islote que anida a varias especies de aves marinas, entre fragatas, piqueros de patas azules y pelicanos. Sin embargo, lo que más predomina en la reserva son sus arrecifes rocosos que lo rodean donde habitan una diversidad de peces e invertebrados destacando a los erizos, estrellas, corales y anemonas; es así que en cada metro de inmersión en el mar, se podrá tener un panorama diverso, con un mundo nuevo de peces de colores, tortugas marinas y una infinidad de invertebrados.

La reserva Marina "El Pelado" tiene valores mínimos y máximo de temperatura del aire que oscilan entre $16-24^{\circ} \mathrm{C}$ y $24-32^{\circ} \mathrm{C}$, respectivamente, teniendo una temperatura promedio interanual de $23,4{ }^{\circ} \mathrm{C}$, sin embargo, eventos como el Fenómeno del Niño, hacen que se presenten mayores valores de temperatura, donde la anomalía puede alcanzar superar $\operatorname{los} 35^{\circ} \mathrm{C}$, o incluso ser lo puesto con eventos como La Niña que alcanza temperaturas de $-2^{\circ} \mathrm{C}$ (GAD Santa Elena, 2014; MAE, 2013).

La biodiversidad de la región marino - costera y terrestre es muy variada en sus diferentes ecosistemas, existiendo una infinidad de especies de flora y fauna, de macro y micro organismos desde un pequeño estero hasta la riqueza megadiversa de la Cordillera Chongón Colonche, sin embargo, predominan tres ecosistemas con mayor relevancia e importancia económica y ecológica: ecosistema marino-costero, manglar, y bosque deciduo del tipo seco. Las principales causas de degradación que afectan a los ecosistemas son las actividades humanas de producción económica, vinculadas a la caza, pesca y turismo, además de los efectos de cambio climático que ha traído consecuencias en el ciclo hídrico y de estaciones lluviosas y secas de manera anormal (GAD Santa Elena, 2014).

El Refugio de Vida Silvestre Marino Costero Pacoche (Figura 1c) está ubicada en el cabo de San Lorenzo al sur de Manta, Provincia de Manabí, debe su nombre a las colinas de Pacoche y limita con varios asentamientos humanos en la línea costera como San Lorenzo, Río Calas, Las Piñas y Santa Rosa, dentro de la reserva se encuentran los asentamientos 
(a

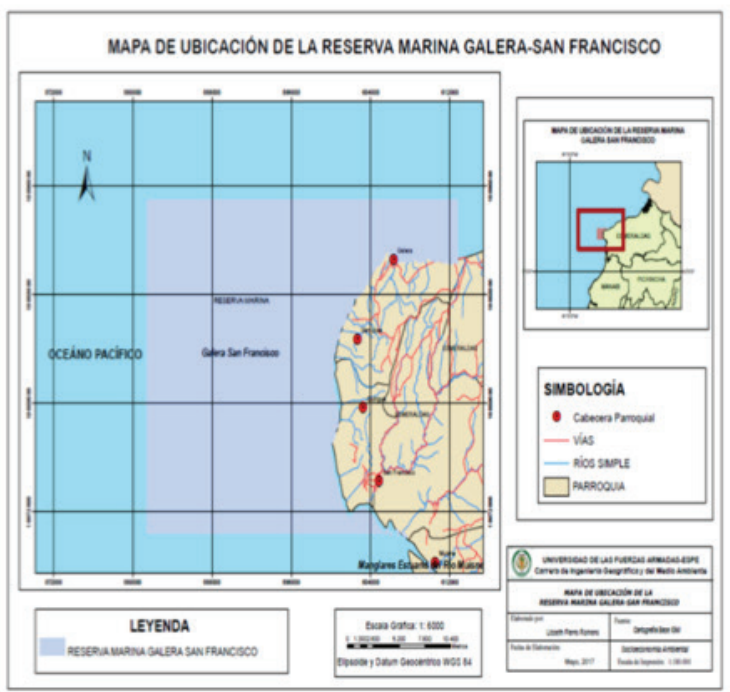

(c

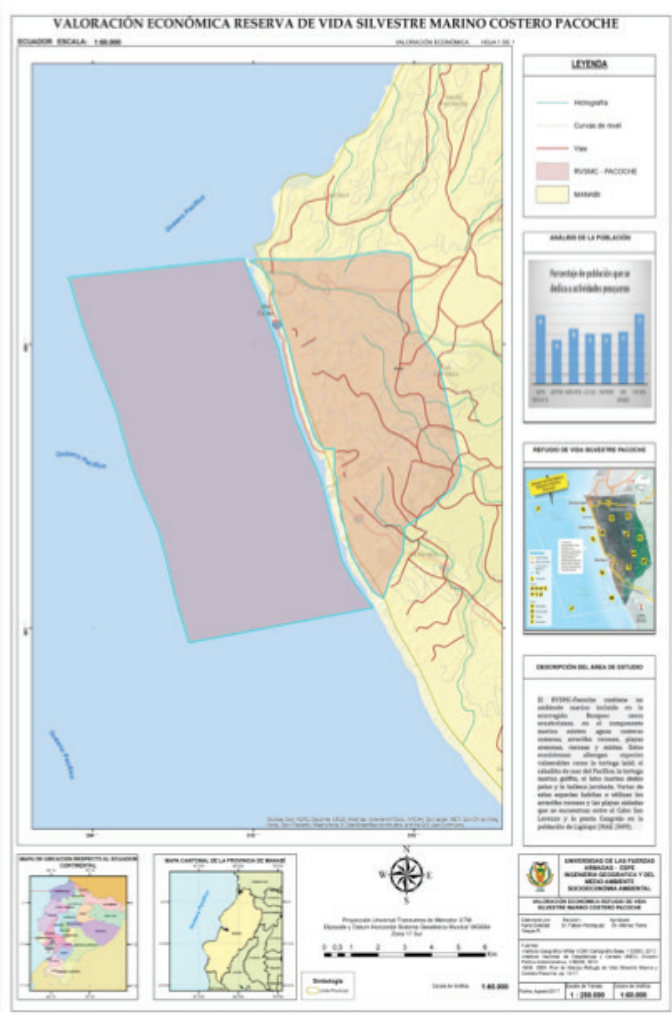

(b

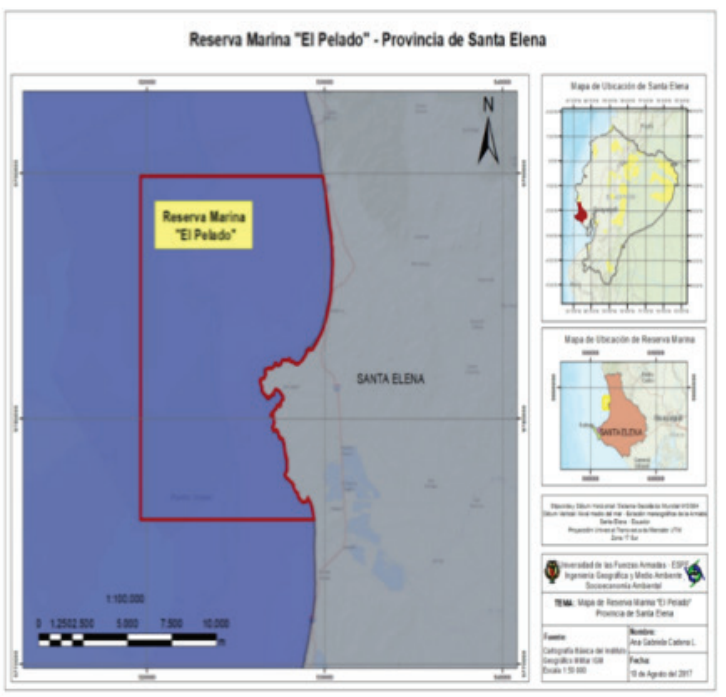

(d

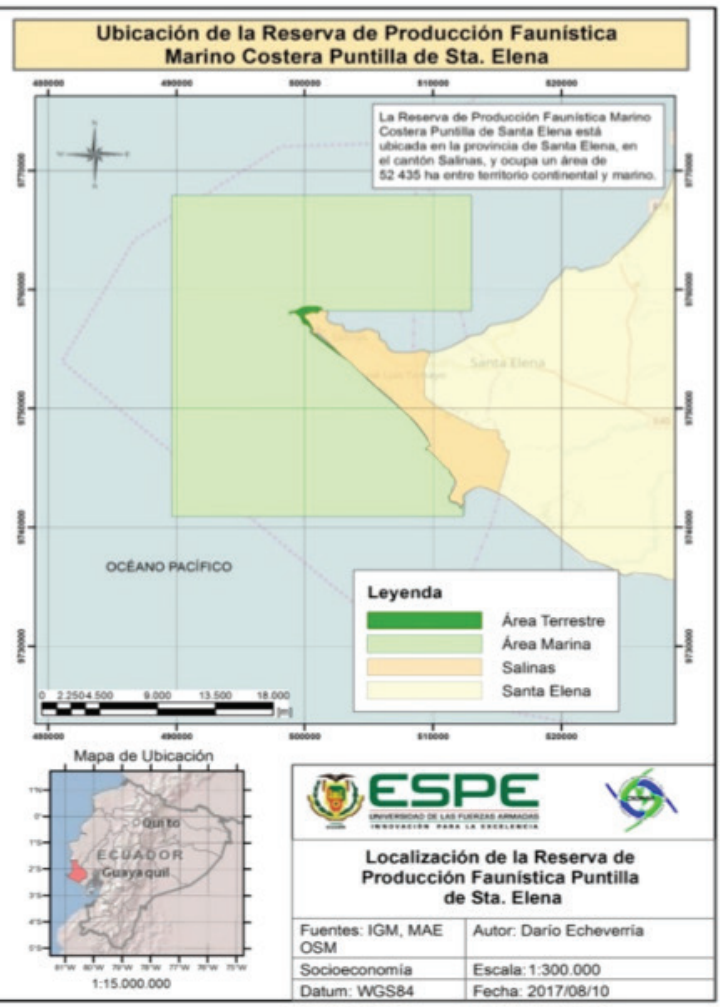

Figura 1. Ubicación de las Áreas Marino-Costeras Protegidas: (a) Reserva Marina Galera-San Francisco, (b) Reserva Marina "El Pelado", (c) Reserva de Vida Silvestre Marino-Costero Pacoche, (d) Reserva de Producción Faunística Marino-Costera Puntilla de Santa Elena. 
La Solita y Agua Fría (MAE, 2009). El refugio tiene un total de 13.545 hectáreas de las cuales 5.045 ha. corresponden a formaciones vegetales de bosque seco tropical y 8.500 ha. de zona marina (MAE, 2009).

El refugio se ubica en un clima tipo tropical megatérmico árido a semiárido con temperaturas medias anuales de $24^{\circ} \mathrm{C}$ y precipitaciones inferiores a los $500 \mathrm{~mm}$ con una sola estación lluviosa entre enero y abril. En el sistema marino-costero del Refugio se encuentras varias comunidades de especies marinas que ocupan aguas someras, los arrecifes rocosos y los $13 \mathrm{~km}$ de playas arenosas y rocosas (MAE, 2009).

La Reserva de Producción Faunística Marino Costera Puntilla de Santa Elena tiene una extensión de 52.435,19 hectáreas, de las cuales 52.231,37 ha. corresponden a la superficie marina, y 203,82 ha. a la superficie terrestre o costera (Figura 1d). La reserva se ubica en el cantón Salinas y es el punto más extremo de la costa continental de América del Sur y separa la bahía de Santa Elena del Golfo de Guayaquil. Las aguas de la reserva son la fuente de subsistencia para varias poblaciones pesqueras y juegan un papel importantísimo en la protección y recuperación de peces que han disminuido por la sobrepesca a lo largo de los años. Aparte del área marina, la reserva incluye playas, acantilados y una pequeña extensión de matorrales y bosques secos del litoral (MAE, 2015b).

El área marina no es muy profunda $(30-50 \mathrm{~m})$ y recibe suficiente luz solar, lo que genera una abundancia de fitoplancton y zooplancton, microorganismos que constituyen la base de la cadena alimenticia en los océanos. El clima en esta reserva, y en general en toda la Península de Santa Elena, es seco y presenta una vegetación típica de zonas áridas. Esto se debe a la influencia de la corriente fría de Humboldt que al estar cerca de estas costas ocasiona una reducción de la evaporación de las aguas oceánicas y, consecuentemente, genera menos nubes y lluvias (MAE, 2015b). El área protegida se encuentra dentro de las ecoregiones de Guayaquil (área marina), y la de Bosques Secos de Ecuador (área terrestre), de donde la zona terrestre se encuentra en la provincia biogeográfica árida del país, y la zona marina, en la provincia del Pacífico Tropical Oriental. Además, los ecosistemas que constan en la REMACOPSE son aguas costeras, arrecifes coralinos, arrecifes rocosos, playas de arena, playas mixtas (arena y roca), acantilados, matorral seco de tierras bajas, matorral seco litoral y espinar litoral (MAE \& MDN, 2009).

Debido a su riqueza marina, existen muchas poblaciones y asentamientos humanos que se dedican principalmente a la pesca artesanal que viven dentro y cercanas a las reservas marinas analizadas. La pesca artesanal es uno de los principales medios para subsistir y es una actividad económica de tradición e historia. El sector marítimo de interés para el Ecuador sustenta pesquerías importantes de carácter pelágico y demersales y particularmente el sector estuarino ofrece un ambiente favorable para la actividad acuícola. Ambas se constituyen en un grupo industrial para el Ecuador en el cual se emplean alrededor de 120.000 personas (FAO, 2003).

Según la FAO, (2003), la pesca extractiva es una actividad orientada a la pesca de 
poblaciones de peces transzonales y altamente migratorias (atunes principalmente), a las poblaciones de peces pelágicos pequeños (enlatados y harina de pescado), especies demersales como pesca acompañante y a la pesca del camarón marino. Para la pesca artesanal, el grupo de peces pelágicos es uno de los más representativos en cuanto a ingresos. Además se tiene también la pesca blanca, que Incluye especies como pargo, atún, corvina, dorado, róbalo, picudo, huayaipe cuyas poblaciones se ubican en zonas costeras. El Instituto Nacional de Pesca estima que los desembarques totales promedio de este subsector alcanza entre las 30000 y $70000 \mathrm{TM} / \mathrm{año}$, de los cuales el $63 \%$ corresponden a pelágicos, el $29 \%$ a peces demersales y el restante $8 \%$ a otras especies (FAO, 2003).

Los desembarques artesanales abastecen principalmente al mercado interno para el consumo de pescado y mariscos frescos. Los desembarques de la flota industrial, en su mayor parte, se destinan a la exportación de pescado fresco, congelado, enlatados y harina, aunque estos dos últimos se comercializan también en el país. Lo propio sucede con la pesca de cultivo, especialmente camarón, no así la trucha, cuyo mayor mercado es el nacional (FAO, 2003).

Como un sector dentro de la pesca extractiva, la pesca artesanal comprende una amplia gama de modalidades que van desde la ancestral recolección a mano de mariscos hasta el uso de embarcaciones motorizadas que operan en aguas soneras y en mar abierto. Su característica básica es la operación manual de las artes de pesca. En el Ecuador se distinguen dos clases de pesca artesanal: la marítima continental, y la de Galápagos. Para el primer grupo antes mencionado, se tienen las opciones pesca de recolección, pesca artesanal costera y oceánica. (FAO, 2008).

El sector pesquero de la provincia de Santa Elena, por ejemplo, constituye un motor clave en la actividad productiva de la región. La pesca, caracterizada por un mercado competitivo con márgenes de utilidad elevados, genera rentas desiguales para los actores involucrados en esta actividad productiva; la gran empresa es, generalmente, la que capta los mayores beneficios, especialmente, por el dominio de estrategias de comercialización que le permiten asegurar alta rentabilidad. Sin embargo, la mediana y pequeña empresa se ve limitada por una insuficiente estructura organizativa y la desarticulación de sus estrategias comerciales, poniéndolas en riesgo para seguir compitiendo, con posibilidades, incluso de quedar fuera del mercado (Benavides et al., 2014).

Las especies más comunes de peces para las comunidades de pescadores artesanales se resumen en la Tabla 2, y el precio que se paga por libra de pescado tiene un promedio de 1,50 dólares. El precio de los crustáceos, por ejemplo, se paga en el mercado 4,00 dólares por libra al pescador. Los pepinos de mar solo son pescados en la reserva marina La Puntilla de Santa Elena, en las otras tres reservas no se reportaron. Por otro lado, la pesca de langosta y camarón solo se reportaron en la reserva marina Galera-San Francisco. El pulpo fue el único crustáceo que se reportó en todas las cuatro reservas. Existen también muchas especies de peces ornamentales que también se extraen, pero se desconoce el volumen y el precio.

Tabla 2. Especies de peces y crustáceos capturados en las reservas marinas 


\begin{tabular}{|l|l|l|}
\hline \multicolumn{2}{|l|}{ Tipo de pesca } & \multicolumn{2}{|l|}{ Captura por faena (lbs) por embarcación } \\
\hline Peces & escasez & abundancia \\
\hline Dorado & 100 & 1200 \\
\hline Albacora & 100 & 400 \\
\hline Bonito & 100 & 1200 \\
\hline Pargo & 100 & 300 \\
\hline Corvina & 50 & 200 \\
\hline Rabón & 50 & 1000 \\
\hline Carita & & \\
\hline Crustáceos & & \\
\hline Langosta & 10 & 15 \\
\hline Camarón & 4 & 10 \\
\hline Pulpo & 2 & 10 \\
\hline Pepinos de mar & & \\
\hline Funte: Luna\& Campos, $2008 ;$ & MEE \&DN, 2009; GAD Santa Elena, 2014 \\
\hline
\end{tabular}

Fuente: Luna\& Campos, 2008; MAE \& MDN, 2009; GAD Santa Elena, 2014.

Elaboración: Autores

\section{METODOLOGÍA}

Dentro de las reservas marino-costeras se pueden encontrar un sin número de bienes y servicios ambientales, como el recursos pesquero, con diversos ecosistemas tanto superficiales como subterráneos como arrecifes de coral, manglares, pastos marinos y lagunas costeras que proveen un abanico de contribuciones directas e indirectas al bienestar humano y a los sectores económicos de las naciones. Sin embargo, en muchas ocasiones se desconocen la mayoría de estos beneficios. Debido a esto, ha surgido el concepto del Valor Económico Total - VET, como herramienta que busca incluir y esquematizar todos los beneficios de los ecosistemas, teniendo valores biofísicos, económicos y sociales. La valoración económica poder contar con un indicador que muestre su importancia en el bienestar social, que permita compararlo con otros componentes del mercado en un mismo patrón monetario. El objetivo es obtener el valor económico de cada reserva marina basada en los bienes y servicios que provee a las comunidades.

Los beneficios económicos de la existencia del Sistema Áreas Protegidas MarinoCosteras $\left(B_{R M}\right)$ puede ser definido como:

$$
B_{R M}=\sum_{j=1}^{k} \sum_{i=1}^{n} B_{i}^{j} \quad \text { donde } j=1,2,3 \cdots, k
$$


Donde $B_{R M}$ es la estimación de los beneficios económicos de los bienes y servicios ambientales $i$ de las reservas marino-costeras protegidas. Los beneficios económicos de cada una de las reservas pueden ser estimados de la siguiente forma:

$$
\begin{aligned}
& B_{i}^{j}=\int_{n}^{j}\left(X_{i}^{j}\right) \\
& B_{1}^{j}=\int_{1}^{j}\left(X_{1}^{j}\right) \\
& B_{2}^{j}=\int_{2}^{j}\left(X_{2}^{j}\right) \\
& \vdots \\
& B_{k}^{j}=\int_{n}^{j}\left(X_{n}^{j}\right)
\end{aligned}
$$

Los beneficios económicos de dada una de las reservas marino-costeras $B_{i}^{j}$ son a la vez una función de los distintos bienes y servicios ambientales $X$ y se define como:

$$
\begin{gathered}
X_{i}^{j}=\sum_{i=1}^{n}\left(x_{i}\right) \quad \text { donde } i=1,2,3, \cdots, n \\
x_{n}^{i}=\int_{i=1}^{n}\left(x_{1}^{i}\right),\left(x_{2}^{i}\right),\left(x_{3}^{i}\right), \cdots,\left(x_{n}^{i}\right)
\end{gathered}
$$

Donde $X_{i}$ es la sumatoria de los bienes y servicios ambientales $x_{i}$. La sumatoria de los bienes y servicios ambientales $B_{R M}$ representa los beneficios económicos que la presencia de las reservas marino-costeras proporciona al país.

El primer paso es definir los bienes y servicios ambientales a ser evaluados. Los ecosistemas marino-costeros tienen funciones ecosistémicas muy importantes que benefician directa e indirectamente al bienestar humano (Tabla 3). Según la Sociedad Ecológica de América - ESA (2000) estos servicios ecosistémicos son los procesos por los cuales los ecosistemas producen recursos que a menudo los seres humanos los toman por descontados. 
Tabla 3. Bienes y Servicios Ambientales en los Ecosistemas Marino-Costeros

\begin{tabular}{|c|c|c|c|c|c|}
\hline Servicio Ecosistémico & Manglares & $\begin{array}{l}\text { Arrecifes } \\
\text { Coralinos }\end{array}$ & Pastos Marinos & $\begin{array}{l}\text { Océano } \\
\text { Abierto }\end{array}$ & Lagunas costeras \\
\hline $\begin{array}{l}\text { Producción de } \\
\text { alimento }\end{array}$ & $\checkmark$ & $\checkmark$ & $\checkmark$ & $\checkmark$ & $\checkmark$ \\
\hline $\begin{array}{l}\text { Protección costera } \\
\text { contra la erosión }\end{array}$ & $\checkmark$ & $\checkmark$ & $\checkmark$ & & \\
\hline $\begin{array}{l}\text { Protección contra } \\
\text { fenómenos naturales }\end{array}$ & $\checkmark$ & $\checkmark$ & & & $\checkmark$ \\
\hline $\begin{array}{l}\text { Purificación del } \\
\text { agua, absorción de } \\
\text { contaminantes }\end{array}$ & $\checkmark$ & $\checkmark$ & $\checkmark$ & $\checkmark$ & $\checkmark$ \\
\hline Captura de carbono & $\checkmark$ & $\checkmark$ & $\checkmark$ & $\checkmark$ & $\checkmark$ \\
\hline $\begin{array}{c}\text { Almacenamiento de } \\
\text { carbono }\end{array}$ & $\checkmark$ & $\checkmark$ & $\checkmark$ & $\checkmark$ & $\checkmark$ \\
\hline $\begin{array}{c}\text { Hábitat de } \\
\text { biodiversidad }\end{array}$ & $\checkmark$ & $\checkmark$ & $\checkmark$ & $\checkmark$ & $\checkmark$ \\
\hline Turismo & $\checkmark$ & $\checkmark$ & $\checkmark$ & $\checkmark$ & $\checkmark$ \\
\hline $\begin{array}{l}\text { Producción de } \\
\text { materias primas }\end{array}$ & $\checkmark$ & $\checkmark$ & $\checkmark$ & $\checkmark$ & $\checkmark$ \\
\hline $\begin{array}{c}\text { Protección y aporte a } \\
\text { las condiciones aptas } \\
\text { para ecosistemas } \\
\text { marinas }\end{array}$ & $\checkmark$ & $\checkmark$ & $\checkmark$ & $\checkmark$ & $\checkmark$ \\
\hline Regulación del clima & $\checkmark$ & $\checkmark$ & $\checkmark$ & $\checkmark$ & $\checkmark$ \\
\hline Recursos Genéticos & $\checkmark$ & $\checkmark$ & $\checkmark$ & $\checkmark$ & $\checkmark$ \\
\hline $\begin{array}{c}\text { Soporte para el } \\
\text { transporte marítimo }\end{array}$ & & & & $\checkmark$ & \\
\hline $\begin{array}{l}\text { Ciclo de nutrientes, } \\
\text { aportes y retenciones }\end{array}$ & $\checkmark$ & $\checkmark$ & $\checkmark$ & $\checkmark$ & $\checkmark$ \\
\hline $\begin{array}{l}\text { Refugio de larvas, } \\
\text { sala cuna de peces }\end{array}$ & $\checkmark$ & $\checkmark$ & $\checkmark$ & & $\checkmark$ \\
\hline Producción de energía & $\checkmark$ & & & $\checkmark$ & \\
\hline
\end{tabular}

Fuente: Prato, J, y Reyna, J., 2015. Elaboración: Autores

Del grupo de bienes y servicios ambientales presentes en las reservas marino-costeras, se seleccionan aquellos de los cuales se dispone información de tal forma que se limita el análisis económico de las reservas marinas. Sobre la base de la información recopilada sobre las cuatro reservas, el análisis se limitó a dos servicios ecosistémicos: el almacenamiento de carbono y la producción de alimento. 
Para la valoración de la producción de alimento se analizará mediante la metodología de precios de mercado. El precio de mercado es una referencia que señala hasta cuanto es la disposición a pagar por el producto en cuestión y, simultáneamente, hasta desde qué cantidad de dinero los productores estarán dispuestos a recibir para comercializarlo. Esto se explica desde la misma definición como funciona la oferta y demanda (Losano, 2005). Debido a que es una zona protegida, el Ministerio del Ambiente permite realizar solo la pesca de carácter artesanal.

La valoración del servicio ambiental de almacenamiento de carbono se estima a partir de los valores de la biomasa seca obtenidas del bosque seco en las reservas marino-costeras. La superficie del área del bosque y el contenido de carbono se extrapolan a toneladas por hectárea, y así puede tener la medida estándar de almacenamiento de captura de carbono (Lee, et al., 2009). Para su valoración económica se utiliza también el método de costo del daño evitado a nivel global por la mitigación del cambio climático (Jenkins et al., 2010). Este costo consiste en el valor del beneficio social global que origina mitigar el cambio climático, ya que al hacerlo evita probables daños a futuro asociados a riesgos naturales, determinado en base a los modelos de equilibrio de costo social del carbono. Sobre la base de las existencias de carbono y el potencial que éste puede tener en las economías nacionales, el almacenamiento de carbono puede ser definido como:

$$
X_{c}=\sum_{i}^{n} P_{i}^{c} Q_{i}^{c} N_{i}^{c}
$$

Donde

$X_{c}=$ Beneficio por el almacenamiento de carbono (\$/año)

$P_{c}=$ Precio del carbono almacenado, no liberado (\$/tonelada)

$Q_{i}^{c}=$ Cantidad de carbono almacenada ( $\left.\mathrm{t} / \mathrm{ha} / \mathrm{año}\right)$

$N_{i}^{c}=$ Número de hectáreas (ha) reconocidas como fuentes de almacenamiento de carbono $i=$ Tipo de bosque considerado para el almacenamiento de carbono.

La estimación del valor de almacenamiento de carbono se define como:

$V X_{C}=$ Contenido $C$ en bosque $\left(\operatorname{ton} \frac{\mathrm{CO}_{2}}{h a}\right) *$ Costo social de $C\left(\frac{\$}{\operatorname{ton} \mathrm{CO}_{2}}\right)$ 
Para tener una idea más clara de la fórmula propuesta nos basamos en los resultados de campo sobre densidad del suelo y el contenido de carbono orgánico total a una profundidad determinada, definido en (3), donde $\rho$ es la densidad del suelo medido en $(\mathrm{g} / \mathrm{cm} 3)$, h es la profundidad y $\mathrm{C}$ el porcentaje en peso de carbono orgánico en el suelo.

Contenido $C$ bosque seco $\left(\frac{\text { ton } \mathrm{CO}_{2}}{h a}\right)=(\rho * h * C * 100) * \frac{44}{12}$

Logrando de esta manera establecer el valor del servicio ambiental de que la reserva tiene por medio de la valoración de carbono en los bosques secos del área.

Los beneficios de la pesca artesanal en las áreas marino-costeras protegidas y sus zonas de influencia se definen como:

$$
X_{p e}=\sum_{i}^{n} P_{i}^{p e} Q_{i}^{p e}
$$

Donde

$X^{p e}=$ Beneficio por el Aprovechamiento de Pesca artesanal (\$/año)

$P^{p e}=$ Precio de pesca de la especie $i(\$ / \mathrm{t})$

$Q^{p e}=$ Volumen de pesca comercializado de la especie $i(\mathrm{t} / \mathrm{año})$

Para realizar la aproximación cuantitativa a la contribución económica de la pesca artesanal de las áreas marino-costeras ecuatorianas se utilizaron valores de la pesca realizada en canoa utilizando las estadísticas expuestas por el INP (Instituto Nacional de Pesca) y el INEC (Instituto Nacional de Estadística y Censos). Adicionalmente, se utilizó la transferencia de beneficios para valorar la pesca del pepino de mar en la puntilla Santa Elena. El método nos indica que se estiman los valores tomando los valores por unidad promedio provenientes de los datos de otro sitio de estudio. La transferencia de beneficios utiliza la aplicación directa de las estadísticas de una investigación original y las transfiere a un nuevo sitio de estudio. Dentro de estas estadísticas se pueden encontrar datos como medidas de disponibilidad a pagar por persona, medidas de elasticidad a los efectos marginales (Osorio, 2006).

\section{RESULTADOS}

La biodiversidad de la región marino-costera y terrestre es muy variada en sus diferentes ecosistemas, existiendo una infinidad de especies de flora y fauna, de macro y micro organismo que consideran desde un pequeño estero hasta la riqueza megadiverso de la Cordillera Chongón Colonche. La estimación del valor económico de los beneficios de uso directo e indirecto de las reservas marino-costeras del presente estudio fue en base a los precios del mercado. 
La pesca artesanal es uno de los bienes y servicios ambientales que estas reservas proveen. El valor se obtuvo mediante el cálculo del excedente del consumidor, es decir, el precio que recibe cada pescador artesanal por el volumen que captura durante el año de trabajo menos sus costos de producción.

Los resultados obtenidos sobre la información recolectada en las áreas de estudio (Tabla 4) demuestran que el aporte de las reservas marinas es muy importante para las poblaciones de la zona que se dedican a la actividad de pesca artesanal. El total de los beneficios obtenidos por las cuatro áreas marino-costeras protegidas sobre pasa los 100 millones de dólares anuales.

A pesar que no tiene la mayor extensión de zona marina, la reserva Costero-Marina Galera-San Francisco tiene el mayor aporte que las otras zonas marino-costeras. Es importante resaltar que solo se incluyó la pesca artesanal de peces y no se tomó en cuenta los crustáceos como camarones, langostas, pulpos, pepinos de mar y otros debido a que no se obtuvo información sobre los volúmenes de extracción salvo en las parroquias Galera y El Quingue donde se obtuvo valores de pesca para langosta, camarón y pulpo y están incluidos en los resultados para la reserva marino costera Galera San Francisco. Se conoce que existe extracción de pepino de mar y de langosta en la Reserva Puntilla Santa Elena, pero la información no estuvo disponible.

Tabla 4. Beneficios económicos por la actividad pesquera artesanal

\begin{tabular}{|c|c|c|c|c|}
\hline Reserva Marina & Área $\left(\mathrm{km}^{2}\right)$ & Reserva marina (ha) & Pesca (Ton) & Beneficios (US\$) \\
\hline Galera-San Francisco & 540 & $535.010,0$ & 23136,0 & $76.427 .007,48$ \\
\hline El Pelado & 13,101 & $13.050,0$ & 2147,7 & $7.020 .168,56$ \\
\hline La Puntilla de Santa Elena & 52,44 & $52.231,4$ & 2257,2 & $7.382 .277,40$ \\
\hline Pacoche & 13,56 & $1.504,0$ & 3141,52 & $9.267 .783,06$ \\
\hline TOTAL & & & & $\mathbf{1 0 0 . 0 9 7 . 2 3 6 , 5 0}$ \\
\hline
\end{tabular}

Fuente: INP y el INEC (2016); GADs Planes de Desarrollo Territorial. Elaboración: Autores

El beneficio indirecto del almacenamiento de carbono se obtuvo sobre la base de los remanentes de bosques de cada una de las reservas marino costeras (Tabla 5). El valor total por el almacenamiento de $\mathrm{C}$ alcanza 5,3 millones de dólares anuales. El precio de referencia fue el del mercado de carbono del Banco Mundial que está alrededor de los 3,5 \$/Ton de carbono. Se utilizó este precio referencial debido a que el Ecuador califica para este mercado de carbono, no se tomaron en cuenta otros mercados con precios referenciales superiores porque el país no califica o simplemente porque aún se encuentran en su etapa de discusión y desarrollo. 
La Reserva Marino-Costera Galera San Francisco aporta con mayor cantidad debido a su mayor extensión de bosques naturales y a que son bosques muy húmedos tropicales. Por otro lado, la Reserva Marino Costera La Puntilla Santa Elena no presenta ningún beneficio y se debe al hecho que la parte terrestre se limita a playas y acantilados sin mayor presencia de comunidades vegetales y bosques.

Tabla 5. Beneficios económicos por el almacenamiento de $\mathrm{C}$ en las reservas marinas

\begin{tabular}{|c|c|c|c|c|}
\hline Reserva Marina & $\begin{array}{c}\text { Área } \\
\left(\mathrm{km}^{2}\right)\end{array}$ & $\begin{array}{c}\text { Bosque natural } \\
(\mathrm{ha})\end{array}$ & $\begin{array}{c}\text { Almacenamiento } \\
\text { de C TM }\end{array}$ & $\begin{array}{c}\text { Beneficios } \\
\text { (US\$) }\end{array}$ \\
\hline Galera-San Francisco & 540 & 4990 & $1.297 .400,00$ & $4.540 .900,00$ \\
\hline El Pelado & 13,101 & 96 & $13.200,00$ & $46.200,00$ \\
\hline La Puntilla de Santa Elena & 52,44 & 203,82 & - & 0,00 \\
\hline Pacoche & 13,56 & 1504 & $206.800,00$ & $723.800,00$ \\
\hline TOTAL & & & & $\mathbf{5 . 3 1 0 . 9 0 0 , 0 0}$ \\
\hline
\end{tabular}

Fuente: INP y el INEC (2016); GADs Planes de Desarrollo Territorial. Elaboración: Autores

El valor total de los aportes de las cuatro reservas marino-costeras de la costa pacífica del Ecuador de dos bienes y servicios ambientales contabilizados alcanza los 105,41 millones de dólares. Gran parte se obtiene de la pesca artesanal, siendo beneficiarios directos 6.800 familias de la costa pacífica ecuatoriana. El valor de la pesca artesanal es de 14.720,18 dólares anuales, ingresos que son superiores a los 5.968,98 dólares del PIB per cápita del Ecuador para el año 2016.

El aporte económico de las cuatro reservas marino costeras es relevante si lo comparamos con el presupuesto del Estado (PGE) en 2016 y con el PIB de ese año (Tabla 6). Los beneficios económicos de las cuatro reservas marino-costeras representan el $0,35 \%$ y 0,1\% del PIB para el año 2016.

Tabla 6. Beneficios económicos de las reservas marino costeras con relación al PIBH y PGE

\begin{tabular}{|c|c|c|c|}
\hline & $\begin{array}{c}\text { Beneficios (millones } \\
\text { US\$) }\end{array}$ & $\begin{array}{c}\text { BRMC/PGE } \\
\mathbf{2 0 1 6}(\mathbf{\%})\end{array}$ & $\begin{array}{c}\text { BRMC/PIB } \\
\mathbf{2 0 1 6}(\mathbf{\%})\end{array}$ \\
\hline Reservas marino-costeras & 105,41 & & \\
\hline PGE 2016 & $29.835,00$ & 0,35 & - \\
\hline PIB 2016 & $102.426,00$ & - & 0,10 \\
\hline
\end{tabular}

Fuente: BCE, 2016; Banco Mundial, 2016. Elaboración: Autores 


\section{DISCUSIÓN}

Las Reservas Marinas poseen funciones ecosistémicas que suministran bienes y servicios muy importantes a las comunidades costeras que ayudan a su subsistencia. Adicionalmente, estas funciones ecológicas proveen servicios indirectos que incrementan las actividades económicas de las comunidades.

El valor económico total de las reservas marino-costeras aún está por estimarse, sin embargo, el presente trabajo ya demuestra el importante aporte económico que la creación de estas reservas da al país. En el presente estudio solo se tomaron en cuenta solo dos bienes y servicios ambientales, la pesca artesanal y el almacenamiento de carbono de los bosques naturales. El aporte económico de estas cuatro reservas marino-costeras llega a 105,41 millones de dólares, siendo actividad pesca artesanal la que mayor aporta con 100,09 millones (Tabla 4). Este beneficio es directo para las comunidades de pescadores artesanales que viven dentro y cerca de las reservas marino-costeras. El almacenamiento de carbono aporta significativamente menos, apenas 5,3 millones de dólares, sin embargo se debe tomar en cuenta que estas cuatro áreas marino-costeras no tiene grandes extensiones de territorio continental y consecuentemente no existe grandes extensiones de con cobertura vegetal natural, apenas 6.793,82 has (Tabla 5). Por otro lado, el estudio no consideró la captura y almacenamiento de carbono por el océano, ya sea por el aporte de las poblaciones de los sistemas bioacuáticos (algas) o por absorción directa del $\mathrm{CO}_{2}$ por parte del océano que puede ser considerable.

Adicionalmente, el estudio no consideró otros servicios ambientales como recreación y estética, como está descrito en la Tabla 3, a pesar que existe turismo en las cuatro reservas marino-costeras como por ejemplo la observación de las ballenas jorobadas que atrae a miles de turistas. Sin embargo, no existen estudios formales que sirvan de referencia confiable. La mayoría de documentos revisados solo presentan la oferta de estas áreas marino-costeras protegidas, pero no mencionan nada sobre la demanda de estos servicios ambientales.

A pesar de las limitaciones que el estudio ha tenido con relación a la información disponible, las cuatro áreas marino-costeras analizadas si presentan un aporte económico al país. Los valores presentados en la Tabla 6 muestra el aporte importantísimo si lo comparamos con el presupuesto general del estado para el 2016 que fue de 29 mil millones de dólares. El aporte fue de 0,35\% que es significante, como significante es si lo comparamos con el producto interno bruto del país para ese año que fue de $100 \mathrm{mil}$ millones de dólares representando el $0,1 \%$ del PIB.

En definitiva, el estudio muestra que los precios de mercado son una buena herramienta para la estimación del aporte económico de cuatro reservas marino-costeras al país, aporte real que proveen los ecosistemas, ya que, en muchas ocasiones la información de las áreas protegidas se limitan a presentar la parte física y en ocasiones la social de las 
mismas, haciendo que ciertos servicios ecosistémicos parezcan invisibles o no se tomen en cuenta como beneficios que generan a la población.

\section{REFERENCIAS}

Akhter, S., and Yew, T. S. (2013). Economic valuation of marine protected areas: A review of studies in Southeast Asia. The International Journal of Social Sciences, 13 (1): 1-16.

Benavides, A., García, L., Alejandro, C. \& Carcelén F. (2014). El sector pesquero de Santa Elena: análisis de las estrategias de comercialización.

Bindoff, N. L., Willebrand, J., Artale, V., Cazenave, A., Gregory, J. M., et al. (2007). Observations: oceanic climate change and sea level. In Climate Change 2007: The Physical Science Basis: Contribution of Working Group I to the Fourth Assessment Report of the Intergovernmental Panel on Climate Change, ed. S Solomon, D Qin, M Manning, Z Chen, M Marquis, et al., Cambridge: Cambridge Univ. Press. pp. 385-432.

Doney, S. C., Ruckelshaus, M., Duffy, J. E., Barry, J. P., Chan, F., English, C. A., Galindo, H. M., Grebmeier, J. M., Hollowed, A. B., Knowlton, N., Polovina, J., Rabalais, N. N., Sydeman, W. J., and Talley L. D. (2012). Climate Change Impacts on Marine Ecosystems. Annual Review of Marine Science, 4:11-37, Annual Reviews, Palo Alto. DOI:10.1146/annurevmarine-041911-111611

Doney, S. C., Fabry, V. J., Feely, R.A., and Kleypas J. A. (2009). Ocean acidification: the other CO2 problem. Annual Review of Marine Science, 1:169-192

Fundación Futuro Latinoamericano. (2011). Gobernanza en las Áreas Protegidas Marinas y Costeras: el caso del Ecuador. Quito, Ecuador. $40 \mathrm{p}$.

GAD Cantonal Santa Elena. (2014). Plan de desarrollo y Ordenamiento Territorial - Cantón Santa Elena, Santa Elena, Ecuador.

GESAMP (IMO/FAO/UNESCO-IOC/UNIDO/WMO/IAEA/UN/UN Environment/UNDP Joint Group of Experts on the Scientific Aspects of Marine Environmental Protection). (2018). The Magnitude and Impacts of Anthropogenic Atmospheric Nitrogen Inputs to the Ocean. Rep. Stud. GESAMP No. 97/GAW Report No. 238, 47 p.

GESAMP/IMO/FAO/UNESCO-IOC/UNIDO/WMO/IAEA/UN/UNEP Joint Group of Experts on the Scientific Aspects of Marine Environmental Protection (2015). Pollution in the Open Oceans 2009-2013 - A report by a GESAMP Task Team. In: Boelens, R. and Kershaw, P.J., (eds.). GESAMP Rep. Stud. No. 91, 87 pp.

Hanley, N., Hynes, S., Patterson, D., and Jobstvogt, N. (2006). Economic Valuation of Marine and Coastal Ecosystems: Is it currently fit for purpose? Journal of Ocean and Coastal Economics, 2 (1): 1-24. DOI: 10.15351/2373-8456.1014

Jenkis, W., Murray, B., Kramer, R., and Faulkner, S. (2010).Valuing ecosystem services from wetlands restoration in the Mississippi Alluvial Valley. Ecological Economics, 69 (5): 1051-1061.

Lee, J., Hopmans, D., Rolsto, S. B., y Six., J. (2009). Determining soil carbon stock changes: Simple bulk density corrections fail. Agriculture, California. Agriculture, Ecosystems and Environment, 134 (3-4): 251-256.

Losano, P. M. (2005). Valoración económica de las áreas protegidas costeras desde el punto de vista de los patrimonios intangibles. En Anuario 2004. Trelew, Argentina: Universidad Nacional de la Patagonia San Juan Bosco, Facultad de Ciencias Económicas.

Luna, S. y F. Campos. 2008. Estudio de Alternativas de Manejo para la declaración de la Reserva 
Marina Galera-San Francisco. Ministerio del Ambiente, Conservación Internacional, The Nature Conservancy y Corporación Instituto NAZCA de Investigaciones Marinas. Quito. Ministerio del Ambiente MAE. (2015a). Sistema Nacional de áreas pprotegidas del Ecuador SNAP, Ecuador.

Ministerio delAmbiente MAE. (2015b). Sistema nacional de áreas protegidas del Ecuador. Reserva de Producción de Fauna Marino Costera Puntilla de Santa Elena. Recuperado de: http:// areasprotegidas.ambiente.gob.ec/es/areas-protegidas/reserva-de-producci $\% \mathrm{C} 3 \% \mathrm{~B} 3 \mathrm{n}$ faun $\% \mathrm{C} 3 \%$ ADstica-marino-costera-puntilla-de-santa-elena

Ministerio del Ambiente MAE. (2013). Reserva Marina El Pelado, Santa Elena. Ministerio del Ambiente del Ecuador, Sistema Nacional de Áreas Protegidas, Quito, Ecuador.

Ministerio del Ambiente MAE. (2009). Plan de Manejo Refugio de Vida Silvestre Marino y Costera Pacoche. Ministerio del Medio Ambiente del Ecuador, Sistema Nacional de Áreas Protegidas, Quito, Ecuador.

Ministerio del Ambiente MAE, Ministerio de Defensa Nacional MDN. (2009). Reserva De Producción Faunística Marino Costera La Puntilla de Santa Elena. Plan de Manejo. Ministerio del Ambiente.

Ministerio del Ambiente MAE. (2006). Sistema nacional de áreas protegidas del Ecuador. Recuperado de: http://areasprotegidas.ambiente.gob.ec/es/info-snap

NAZCA. (2006). Análisis de vacíos e identificación de Áreas Prioritarias para la Conservación de la Biodiversidad Marino-Costera en el Ecuador Continental. Resumen Ejecutivo. Ministerio del Ambiente. Quito.

OECD. (2016), Marine Protected Areas: Economics, Management and Effective Policy Mixes. OECD Policy Highlights, OECD Publishing, Paris. http://dx.doi. org/10.1787/9789264276208-en

Organización de las Naciones Unidas para la Agricultura y Alimento FAO. (2016). The State of World Fisheries and Aquaculture, Food and Agriculture Organization, Rome.

Organización de las Naciones Unidas para la Agricultura y Alimento FAO. (2003). Perfil pesquero nacional. Ecuador. Recuperado de: http:/www.fao.org/fi/oldsite/FCP/es/ECU/profile.htm

Organización de las Naciones Unidas para la Agricultura y Alimento FAO. (2008). Información sobre la ordenación pesquera: la república del ecuador. Recuperado de: http://www.fao. org/fi/oldsite/FCP/es/ECU/BODY.HTM

Osorio, J. (2006). El método de transferencia de beneficios para la valoración económica de servicios ambientales: estado del arte y aplicaciones. Semestre económico - Universidad de Medellín.

Peralta, M. (2001). Desembarques de la pesca artesanal ecuatoriana durante el año 2001. Instituto Nacional de Pesca. Guayaquil.

Prato J, y Reyna J. (2015). Aproximación a la valoración económica de la zona marina y costera del Caribe colombiano. Secretaría Ejecutiva de la Comisión Colombiana del Océano. Bogotá, 184 pp.

Rodriguez-Espinosa, F., Toulkeridis, T., Salazar-Martínez, R., Cueva-Giron, J., Taipe-Quispe, A., Vernaza-Quiñonez, L., Padilla-Almeida, O., Mato, F., Cruz-D’Howitt, M., Parra, H., Sandoval, W., Rentería, W. (2017). Economic evaluation of recovering natural protection with concurrent relocation of the population threatened by tsunami hazards in Central Coast Ecuador. Science of Tsunami Hazards, 36 (4): 293-306.

Sala, E., Costella, C., Dougherty, D., Heal, G., Kellher, K., Murray, J. H., Rosenberg, A. A., and 
Sumaila, R. (2013). A General Business Model for Marine Reserves. PLOS One, 8 (4): e58799. doi:10.1371/journal.pone.0058799

Salgado, M. (2016). Análisis de la valoración económica para la conservación de la Reserva de Producción de Fauna Marino Costera Puntilla de Santa Elena. Universidad Católica de Santiago de Guayaquil, Facultad de Ciencias Económicas y Administrativas.

Salm, R.V., Clark, J., and Siirila, E. (2000). Marine and Coastal Protected Areas: A guide for planners and managers. IUCN. Washington DC. xxi + 371pp.

Sanchirico, J. N., Cochran, K. A., and Emerson, P. M. (2002). Marine Protected Areas: Economic and Social Implications. Resources for the Future, Discussion Paper 02-26, Washington D. C. pp. 24.

Shahidul Islam, M., and Tanaka, M. (2004). Impacts of pollution on coastal and marine ecosystems including coastal and marine fisheries and approach for management: a review and synthesis. Marine Pollution Bulletin, 48 (7-8): 624-649. DOI: 10.1016/j. marpolbul.2003.12.004

Silva, Maynard E., Gately, Ellen M., De silvestre, I., (1986). Bibliographic listing of coastal and marine protected areas : a global survey. Woods Hole Oceanographic Institution Technical Report. DOI:10.1575/1912/7543, https://hdl.handle.net/1912/7543

The Ecological Society of America. (2000). Ecosystem Services: A Primer. Ecological Society of America. www.esa.org.

Vikas, M. and Dwarakish, G. S. (2015). Coastal Pollution: A Review. Aquatic Procedia, 4: 381388. doi: 10.1016/j.aqpro.2015.02.051

Xuan, B. B., and Armstrong, C. W. (2016). Marine reserve creation and interactions between fisheries and capture-based aquaculture: A bioeconomic model analysis. Natural Resource Modeling, 00, 1-15. DOI: 10.1111/nrm.12122 